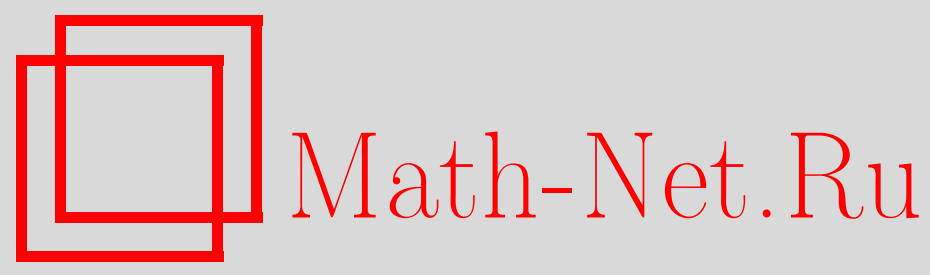

Н. А. Даурцева, О многообразии почти комплексных структур, Матем. заметки, 2005, том 78, выпуск 1, 66-71

DOI: https://doi.org/10.4213/mzm2558

Использование Общероссийского математического портала Math-Net.Ru подразумевает, что вы прочитали и согласны с пользовательским соглашением http://www. mathnet.ru/rus/agreement

Параметры загрузки:

IP: 54.84 .234 .179

26 апреля 2023 г., 10:54:24

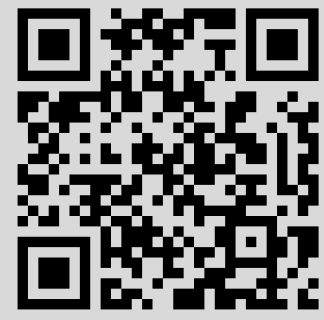




\title{
О МНОГООБРАЗИИ ПОЧТИ КОМПЛЕКСНЫХ СТРУКТУР
}

\author{
Н. А. Даурцева
}

Пусть $\left(M, g_{0}\right)$ - гладкое замкнутое риманово многообразие четной размерности $2 n$, допускающее почти комплексную структуру. В настоящей работе показано, что пространство $\mathscr{A}^{+}$всех почти комплексных структур на $M$, задающих ту же ориентацию, что и фиксированная почти комплексная структура $J_{0}$, является гладким локально тривиальньм расслоением над пространством $\mathscr{A}_{g_{0}}^{+}$ортогональных относительно $g_{0}$ почти комплексных структур, задающих ту же ориентацию, что и $J_{0}$.

Библиограффия: 6 названий.

1. Введение. На симплектическом многообразии $(M, \omega)$ естественно рассматривать такие почти комплексные структуры и римановы метрики, которые согласованы с формой $\omega$. Почти комплексная структура (в дальнейшем ПКС) $J$ на $M$ назьвается $n o$ ложительно ассоциированной с симплектической формой $\omega$, если для любых векторных полей $X, Y$ на $M$ вьполняются условия $\omega(J X, J Y)=\omega(X, Y)$ и $\omega(X, J X)>0$, если $X \neq 0$. Каждая такая ПКС $J$ определяет риманову метрику $g$ на $M$ равенством $g(X, Y)=\omega(X, J Y)$, которая также назьвается ассоииированной.

Обозначим символами $\mathscr{A}_{\omega}^{+}$и $\mathscr{A} \mathscr{M}_{\omega}$ бесконечномерные пространства всех гладких положительно ассоциированных ПКС и, соответственно, ассоциированных метрик на $M$. Формула $g(X, Y)=\omega(X, J Y)$ устанавливает взаимно однозначное соответствие между этими пространствами.

Хорошо известно, что пространство $\mathscr{A} \mathscr{M}_{\omega}$ является ретрактом пространства $\mathscr{M}$ всех римановых метрик. Пусть $g^{\prime} \in \mathscr{M}$ - любая метрика. Существует единственньй кососимметрический автоморфизм $A$ касательного расслоения $T M$ такой, что $\omega(X, Y)=$ $g^{\prime}(A X, Y)$. Тогда легко проверить, что $J=\left(-A^{2}\right)^{-1 / 2} A$ является положительной ассоциированной почти комплексной структурой, а $g(X, Y)=\omega(X, J Y)$ - ассоциированной метрикой, соответствующей структуре $J$.

Приведенная конструкция определяет проекцию $p_{\omega}: \mathscr{M} \rightarrow \mathscr{A} \mathscr{M}_{\omega}, p_{\omega}\left(g^{\prime}\right)=g$. В работе [1] показано, что эта проекция является гладким тривиальньм расслоением, причем слои состоят из римановых метрик, эрмитовых относительно ПКС $J$, соответствующей метрике $g \in \mathscr{A} \mathscr{M}_{\omega}$. В связи с этим результатом естественно поставить вопрос об аналогичном разложении пространства $\mathscr{A}^{+}$всех почти комплексных структур, задающих фиксированную на $M$ ориентацию. Оказывается, что построить проекцию $q_{\omega}: \mathscr{A}^{+} \rightarrow \mathscr{A}_{\omega}^{+}$аналогичным образом, когда слоем является пространство $g$ ортогональных ПКС, не удается. Однако нетрудно заметить, что локально пространство всех почти комплексных структур раскладывается в прямое произведение ортогональных ПКС 
и ассоциированных ПКС. Поэтому можно пытаться построить проекцию пространства $\mathscr{A}^{+}$на пространство $\mathscr{A} \mathscr{O}_{g_{0}}^{+}$ортогональных ПКС относительно фиксированной метрики, слои которой были бы пространствами положительно ассоциированных метрик. Данная работа посвящена построению именно этой проекции. Имеет место следующая

ТЕОремА. Пространство почти комплексных структур $\mathscr{A}^{+}$имеет структуру бесконечномерного расслоения над пространством $\mathscr{A O}_{g_{0}}^{+}$ортогональных относительно $g_{0}$ почти комплексных структур. При әтом слоем над ортогональной ПКС структурой $J$ является множество всех почти комплексных структур, положстельно ассоциированных с невырожденной формой $\omega_{J}(X, Y)=g_{0}(J X, Y)$.

Идея доказательства заключается в следующем. Рассмотрим сначала проекцию пространства $\mathscr{A}^{+}$на пространство невырожденных кососимметрических 2-форм $\Gamma\left(\Lambda^{2} M\right)$ :

$$
\mathscr{A}^{+} \rightarrow \Gamma\left(\Lambda^{2} M\right), \quad J \rightarrow \omega_{J}, \quad \text { где } \omega_{J}(X, Y)=\frac{1}{2}\left(g_{0}(J X, Y)-g_{0}(X, J Y)\right) .
$$

Затем с каждой невырожденной 2-формой $\omega_{J}$ свяжем ортогональную ПКС $J_{1}$ по формуле $J_{1}=\left(-B^{2}\right)^{-1 / 2} B$, где $B$ такой эндоморфизм, что $\omega_{J}(X, Y)=g_{0}(B X, Y)$.

Этот результат развивает известньй факт о стягиваемости $G L^{+}(2 n, \mathbb{R}) / G L(n, \mathbb{C})$ на $S O(2 n) / U(n)[2]$.

2. Необходимые понятия и факты. Пусть $M$ - гладкое замкнутое многообразие размерности $2 n$. Почти комплексной структурой на $M$ называется поле эндоморфизмов $J_{x}: T_{x} M \rightarrow T_{x} M$, гладко зависящее от $x \in M$ и удовлетворяющее следующему свойству $J_{x}^{2}=-\operatorname{Id}_{x}$, где $\operatorname{Id}_{x}$ - тождественный эндоморфизм $T_{x} M$.

Предположим, что $M$ почти эрмитово, т.е. на нем задана ПКС $J_{0}$ и риманова метрика $g_{0}$, инвариантная относительно $J_{0}$. Фундаментальная форма $\omega$ определяется равенством $\omega(X, Y)=g_{0}\left(J_{0} X, Y\right)$ для любой пары векторных полей $X, Y$, касательных к $M$.

Пусть $\operatorname{End}(T M)$ - расслоение эндоморфизмов касательного расслоения $T M$. Символ Г будет обозначать пространство гладких сечений расслоений. В частности, $\Gamma(\operatorname{End}(T M))$ - пространство гладких сечений эндоморфизмов расслоения $T M$. Пусть

$$
\mathscr{A}=\left\{J \in \Gamma(\operatorname{End}(T M)): J^{2}=-\mathrm{Id}\right\}
$$

- пространство всех гладких ПКС на $M$, а $\mathscr{A}^{+}-$пространство всех $J \in \mathscr{A}$, задающих ту же ориентацию на $M$, что и $J_{0}$. Пространство $\mathscr{A}$ является гладким бесконечномерным многообразием $\Phi$ реше. Касательное пространство $T_{J} \mathscr{A}$ состоит из всех эндоморфизмов $P \in \Gamma(\operatorname{End}(T M))$, антикоммутируюших с $J: P J=-J P$. Eсли $\operatorname{End}_{A J}(T M)-$ расслоение эндоморфизмов, антикоммутирующих с $J$, то $T_{J} \mathscr{A}=\Gamma\left(\operatorname{End}_{A J}(T M)\right)$. Для параметризации $\mathscr{A}$ воспользуемся следующей известной конструкцией. Пусть $P \in$ $\Gamma\left(\operatorname{End}_{A J}(T M)\right)$ и $1-P$ обратим. Координатные карты на $\mathscr{A}$ в окрестности $U(J)=$ $\left\{J_{1} \in \mathscr{A}: 1-J_{1} J-\right.$ изоморфизм $\left.T M\right\}$ элемента $J \in \mathscr{A}$ определяются следующим образом:

$$
\Phi: \Gamma\left(\operatorname{End}_{A J}(T M)\right) \rightarrow \mathscr{A}, \quad P \rightarrow J(1+P)(1-P)^{-1} .
$$


Форма $\omega$ позволяет выделить в $\mathscr{A}$ следующее подмножество:

$$
\mathscr{A}_{\omega}=\{J \in \mathscr{A}: \omega(J X, J Y)=\omega(X, Y) \forall X, Y \in \Gamma(T M)\}
$$

В зависимости от знакоопределенности квадратичной формы $j(X)=\omega(X, J X)$ множество $\mathscr{A}_{\omega}$ распадается на три подмножества $\mathscr{A}_{\omega}^{+}, \mathscr{A}_{\omega}^{-}, \mathscr{A}_{\omega}^{ \pm}$всех $J \in \mathscr{A}_{\omega}$, для которых $j$ определена положительно, отрицательно и знаконеопределена, соответственно. Множество $\mathscr{A}_{\omega}^{+}$представляется наиболее интересным, так как каждая ПКС $J \in \mathscr{A}_{\omega}^{+}$единственньм образом связана с римановой метрикой $g_{J}(X, Y)=\omega(X, J Y)$, т.е. $\mathscr{A}_{\omega}^{+}$связано с пространством ассоциированных метрик $\mathscr{A} \mathscr{M}_{\omega}[3]$.

Посредством конструкции (1) на $\mathscr{A}_{\omega}^{+}$также вводится структура многообразия [4]. В качестве модельного пространства берется пространство $\Gamma\left(\operatorname{End}_{A J_{0}}^{+}(T M)\right)$ всех гладких эндоморфизмов $T M$, антикоммутирующих с $J_{0}$ и симметричных относительно $g_{0}$. Тогда $T_{J} \mathscr{A}_{\omega}^{+}=\Gamma\left(\operatorname{End}_{A J}^{+}(T M)\right)$.

Наличие римановой структуры $g_{0}$ позволяет выделить в $\mathscr{A}(M)$ класс ортогональных $\Pi \mathrm{\Pi K}$ :

$$
\mathscr{A} \mathscr{O}_{g_{0}}=\left\{J \in \mathscr{A}: g_{0}(J X, J Y)=g_{0}(X, Y) \forall X, Y \in \Gamma(T M)\right\} .
$$

Пространство $\mathscr{A} \mathscr{O}_{g_{0}}$ также является гладким бесконечномерным многообразием ше. Касательное пространство $T_{J} \mathscr{A O}_{g_{0}}$ состоит из всех гладких эндоморфизмов, антикоммутирующих с $J$ и кососимметрических относительно метрики $g_{0}$. Расслоение таких эндоморфизмов обозначим $\operatorname{End}_{A J}^{-}(T M)$. Тогда $T_{J} \mathscr{A}_{g_{0}}=\Gamma\left(\operatorname{End}_{A}{ }_{J}(T M)\right)$. Пространство $\mathscr{A} \mathscr{O}_{g_{0}}$ состоит из пространства $\mathscr{A} \mathscr{O}_{g_{0}}^{+}$ортогональных ПКС, задающих ту же ориентацию, что и $J_{0}$, и из $\mathscr{A} \mathscr{O}_{g_{0}}^{-}$ортогональных ПКС, задающих противоположную ориентацию.

Параметризуем $\mathscr{A}_{g_{0}}^{+}$посредством (1). Отображение $\Phi$ будет гомеоморфизмом, если $\operatorname{det}(1-P)>0$ во всех точках $x \in M$.

3. Доказательство теоремы. Поскольку мы имеем дело с пространствами сечений гладких расслоений, достаточно провести доказательство в произвольной точке $x \in M$. Рассмотрим множество почти комплексных структур в точке, т.е. действующих на $\left(T_{x} M, g(x)\right)$. Выбор ортонормированного базиса в $T_{x} M$ позволяет отождествить $\left(T_{x} M, g(x)\right)$ со стандартным евклидовым пространством $\mathbb{R}^{2 n}$ с координатами $\left(x_{1}, \ldots, x_{n}, y_{1}, \ldots, y_{n}\right)$ и плоской метрикой $g_{0}: d s^{2}=d x_{1}^{2}+\cdots+d x_{n}^{2}+d y_{1}^{2}+\cdots+d y_{n}^{2}$. Пусть также $J_{0}$ и $\omega$ стандартные комплексная структура и симплектическая форма на $\mathbb{R}^{2 n}$. Пространство всех комплексных структур на $\mathbb{R}^{2 n}$ обозначим как $A$. Известно [5], что существует естественное взаимно однозначное соответствие между множеством $A$ и однородным пространством $G L\left(2 n, \mathbb{R}^{2 n}\right) / G L(n, \mathbb{C}), \operatorname{dim} A=(2 n)^{2}-2 n^{2}=2 n^{2}$. Известно также, что $A_{\omega}^{+}=S p(n, \mathbb{R}) / U(n), \operatorname{dim} A_{\omega}^{+}=n^{2}+n$ и $A O_{g_{0}}^{+}=S O(2 n) / U(n)$, $\operatorname{dim} A O_{g_{0}}^{+}=n^{2}-n$. Заметим, что $\operatorname{dim} A=\operatorname{dim} A_{\omega}^{+}+\operatorname{dim} A O_{g_{0}}^{+}$.

Лемма 1. Имеем $A_{\omega}^{+} \cap A O_{g_{0}}^{+}=\left\{J_{0}\right\}$.

ДокаЗАтЕльство. Допустим, что существует $J \in A_{\omega}^{+} \cap A O_{g_{0}}^{+}$, отличная от $J_{0}$; тогда $\exists K \in \Gamma\left(\operatorname{End}_{A J_{0}}^{+}(T M)\right), 1-K^{T} K>0$, такой, что $J=(1-K) J_{0}(1-K)^{-1}$. Тогда принадлежность $J \in A O_{g_{0}}^{+}$дает следующую логическую цепочку:

$$
J^{T} J=1 \Longleftrightarrow J^{T}=-J \Longleftrightarrow K=0 \Longrightarrow J=J_{0}
$$


Возьмем произвольную ассоциированную [4] с $\omega$ риманову метрику $g \in A M_{\omega}$ на $\mathbb{R}^{2 n}$. Пусть $A O_{g}^{+}$- пространство комплексных структур на $\mathbb{R}^{2 n}$, ортогональных относительно выбранного скалярного произведения $g$. Если выбрать $J \in A O_{g}^{+}$, то фундаментальная форма $\omega_{J}(X, Y)=g(J X, Y)$ не совпадает с $\omega$. Пусть $A_{\omega_{J}}^{+}-$пространство комплексных структур на $\mathbb{R}^{2 n}$, инвариантных и положительных относительно $\omega_{J}, A^{+}$- пространство комплексных структур на $\mathbb{R}^{2 n}$, задающих ту же ориентацию, что и $J_{0}$. Тогда $\forall J \in A O_{g}^{+}$имеет место следуюшее разложение:

$$
T_{J} A^{+}=T_{J} A_{\omega_{J}}^{+} \oplus T_{J} A O_{g}^{+}
$$

Возникает естественньй вопрос о возможности представить $A^{+}$в виде расслоения. Другими словами, существует ли проекция

1) $\pi: A^{+} \rightarrow A_{\omega}^{+}$такая, что $\forall J \in A_{\omega}^{+}: \pi^{-1}(J)=A O_{g}^{+}$, где $g(X, Y)=\omega(X, J Y)$, или

2) $\pi: A^{+} \rightarrow A O_{g_{0}}^{+}$такая, что $\forall J \in A O_{g_{0}}^{+}: \pi^{-1}(J)=A_{\omega_{J}}^{+}$, где $\omega_{J}(X, Y)=g_{0}(J X, Y)$ ?

Напомним, что $\forall B \in G L(n, \mathbb{R})$ существует полярное разложение [6] на симметрическую, положительно определенную $S$ и ортогональную $O$ относительно фиксированной стандартной метрики $g$ матрищы: $B=S \cdot O$.

Лемма 2. Для произвольной почти комплексной структуры $J \in A^{+}$существует ортогональная почти комплексная структура $J_{1} \in A O_{g_{0}}^{+}$такая, что $J$ лежит в ее окрестности $J \in U\left(J_{1}\right)$, т.е. существует $K \in \operatorname{End}_{A J_{1}}(T M), \operatorname{det}(1-K)>0$ такой, что $J=(1-K) J_{1}(1-K)^{-1}$.

ДоказАтельство. $\forall J \in A^{+}$существует $B \in G L(2 n, \mathbb{R}), \operatorname{det} B>0$, такой, что $J=B J_{0} B^{-1}$; это значит, что для $J$ найдутся симметрическая положительно определенная матрица $S$ и $O \in S O(2 n)$ такие, что $J=S \cdot O \cdot J_{0} O^{-1} S^{-1}$. Пусть $J_{1}=O J_{0} O^{-1}$ ортогональная ПКС, соответствующая $O \in S O(2 n)$. Представим $S$ как сумму коммутирующей с $J_{1}$ и антикоммутирующей с $J_{1}$ матрищ: $B_{1}=\frac{1}{2}\left(S-J_{1} S J_{1}\right)$ и $B_{2}=\frac{1}{2}\left(S+J_{1} S J_{1}\right)$. Поскольку $S$ и $-J_{1} S J_{1}=J_{1} S J_{1}^{-1}$ положительно определены, то $B_{1}=S-J_{1} S J_{1}$ обратима. Тогда

$$
\begin{aligned}
J & =\left(B_{1}+B_{2}\right) O J_{0} O^{-1}\left(B_{1}+B_{2}\right)^{-1}=\left(1+B_{2} B_{1}^{-1}\right) B_{1} J_{1} B_{1}^{-1}\left(1+B_{2} B_{1}^{-1}\right)^{-1} \\
& =\left[B_{1} J_{1}=J_{1} B_{1}\right]=\left(1+B_{2} B_{1}^{-1}\right) J_{1}\left(1+B_{2} B_{1}^{-1}\right)^{-1} .
\end{aligned}
$$

Таким образом, $J=(1-K) J_{1}(1-K)^{-1}$, где $K=-B_{2} B_{1}^{-1}=-\left(S+J_{1} S J_{1}\right)\left(S-J_{1} S J_{1}\right)^{-1}$, т.е. $J \in U\left(J_{1}\right)$. Лемма доказана.

ЗАмЕчАнИЕ. Поскольку матрица $B$, соответствующая $J$, определяется не однозначно, то $J$ может принадлежать окрестностям различных ортогональных ПКС. В связи с тем, что $A_{\omega_{J_{1}}}^{+} \subset U\left(J_{1}\right)$, возникают вопросы: можно ли среди тех $J_{1} \in A O_{g_{0}}^{+}$, окрестности которых содержат $J$, найти такую, в которой $J \in A_{\omega_{J_{1}}}^{+}$?

Однозначньй выбор такого $J_{1} \in A O_{g_{0}}^{+}$для произвольной ПКС $J \in A^{+}$позволяет осуществить конструкция, аналогичная [1]. Пусть $J \in A^{+}$; ей соответствует невырожденная кососимметрическая 2-форма $\omega_{J}(X, Y)=\frac{1}{2}\left(g_{0}(J X, Y)-g_{0}(X, J Y)\right)$. Она невырождена, так как является фундаментальной для метрики $g(X, Y)=\frac{1}{2}\left(g_{0}(X, Y)+\right.$ $\left.g_{0}(J X, J Y)\right)$. Форма определяет кососимметрический эндоморфизм $B: \omega_{J}(X, Y)=$ 
$g_{0}(B X, Y)$. Определим почти комплексную структуру $J_{1}=\left(-B^{2}\right)^{-1 / 2} B$. Она ортогональна относительно $g_{0}$ :

$$
g_{0}\left(\left(-B^{2}\right)^{-1 / 2} B X,\left(-B^{2}\right)^{-1 / 2} B Y\right)=g_{0}\left(X,-B\left(-B^{2}\right)^{-1 / 2}\left(-B^{2}\right)^{-1 / 2} B Y\right)=g_{0}(X, Y)
$$

и задает ту же ориентацию, что и $J_{0}$. Сопоставим $J_{1}$ фундаментальную форму $\omega_{J_{1}}(X, Y)$ $=g_{0}\left(J_{1} X, Y\right)$. Поскольку $J$ и $B^{2}$ коммутируют, из этого следует, что $J$ лежит в $A_{\omega_{J_{1}}}^{+}$:

$$
\begin{aligned}
\omega_{J_{1}}(J X, J Y) & =g_{0}\left(B\left(-B^{2}\right)^{-1 / 2} J X, J Y\right)=\omega_{J}\left(\left(-B^{2}\right)^{-1 / 2} J X, J Y\right) \\
& =\frac{1}{2}\left(g_{0}\left(-\left(-B^{2}\right)^{-1 / 2} X, J Y\right)+g_{0}\left(J\left(-B^{2}\right)^{-1 / 2} X, Y\right)\right)=\omega_{J_{1}}(X, Y) .
\end{aligned}
$$

Таким образом, существует проекция $\pi: A^{+} \rightarrow A O_{g_{0}}^{+}$такая, что $\pi(J)=J_{1} \in A O_{g_{0}}^{+}$и $J \in A_{\omega_{J_{1}}}^{+}$для любого $J \in A^{+}$. Обратно, если $J \in A_{\omega_{J_{1}}}^{+}$для некоторого $J_{1} \in A O_{g_{0}}^{+}$, то $\exists K \in \operatorname{End}_{A J_{1}}^{+}(T M), 1-K^{T} K>0$, такой, что $J=(1-K) J_{1}(1-K)^{-1}$. Тогда

$$
\omega_{J}(X, Y)=g_{0}\left(\frac{1}{2}\left((1-K) J_{1}(1-K)^{-1}+(1-K)^{-1} J_{1}(1-K)\right) X, Y\right),
$$

поэтому соответствующий $J$ эндоморфизм

$$
\begin{gathered}
B=\frac{1}{2}\left((1-K) J_{1}(1-K)^{-1}+(1-K)^{-1} J_{1}(1-K)\right), \\
\left(-B^{2}\right)^{-1 / 2}=2\left((1-K)(1+K)^{-1}+(1-K)^{-1}(1+K)\right)^{-1} .
\end{gathered}
$$

Поскольку для указанного $B$ имеет место равенство $\left(-B^{2}\right)^{-1 / 2} B=J_{1}$, то почти комплексная структура $J$ из $A_{\omega_{J_{1}}}^{+}$проектируется именно на $J_{1}$. Это значит, что слоем расслоения $\left(A, A O_{g_{0}}^{+}, \pi\right)$ является $A_{\omega_{J_{1}}}^{+}$.

ПРЕДЛОЖЕНИЕ. Пространство $A^{+}$является гладким локально тривиальным расслоением над $A O_{g_{0}}^{+}$. Слоем над әлементом $J \in A O_{g_{0}}^{+}$служит пространство $A_{\omega_{J}}^{+}$.

ДокАЗАТЕЛЬСТво. В силу изложенных вьше соображений достаточнопоказать локальную тривиальность. Пусть $U O(J)=U(J) \cap A O_{g_{0}}^{+}$; тогда будем рассматривать $\cup_{J \in A O_{g_{0}}^{+}} U O(J)$ в качестве покрытия $A O_{g_{0}}^{+}$. Окрестность $U O(J)$ параметризуется $\operatorname{End}_{A}{ }_{J}(T M)$. В качестве карт можно использовать следующие отображения:

$$
\begin{gathered}
\varphi_{J}: \operatorname{End}_{A J}^{-}(T M) \times A_{\omega_{J}}^{+} \rightarrow A^{+}, \quad\left(K, J_{1}\right) \rightarrow J_{2}, \\
J_{2}=\left(1-K^{2}\right)^{-1 / 2}(1-K) J_{1}(1-K)^{-1}\left(1-K^{2}\right)^{1 / 2} .
\end{gathered}
$$

Легко проверить, что почти комплексная структура $J_{2}$ лежит в $A_{\omega_{J^{\prime}}}^{+}$, где $J^{\prime}=$ $(1-K) J(1-K)^{-1}$. Поэтому слой $A_{\omega_{J}}$ отображается на $A_{\omega_{J}}$. Обратно, произвольной $J_{2} \in A_{\omega_{J^{\prime}}}$ соответствует $J_{1}=\left(1-K^{2}\right)^{1 / 2}(1-K)^{-1} J_{2}(1-K)\left(1-K^{2}\right)^{-1 / 2} \in A_{\omega_{J}}^{+}$, где $K$ соответствует $J_{1}$ в $U O(J)$. Допустим, что $J^{\prime}$ лежит в пересечении двух окрестностей $U O(J)$ и $U O(\tilde{J})$, т.е. существует $K \in \operatorname{End}_{A}^{-}$такой, что $J^{\prime}=(1-K) J(1-K)^{-1}$, 
и существует $P \in \operatorname{End}_{A \tilde{J}}^{-}(T M)$ такой, что $J^{\prime}=(1-P) \tilde{J}(1-P)^{-1}$. Тогда формулы перехода от $P$ к $K$ имеют вид

$$
P=\left(1-(1-K)(1+K)^{-1} J \tilde{J}\right)^{-1}\left(1+(1-K)(1+K)^{-1} J \tilde{J}\right) .
$$

Если $J_{1} \in A_{\omega_{J}}^{+}$и $\tilde{J}_{1} \in A_{\omega_{\tilde{J}}}^{+}$соответствуют одному и тому же элементу $J_{2} \in A_{\omega_{J}^{\prime}}^{+}$, т.е. $\varphi_{J}\left(K, J_{1}\right)=\varphi_{\tilde{J}}\left(P, \tilde{J}_{1}\right)$, то они согласованы следующим образом:

$\tilde{J}_{1}=(1-P)^{-1}\left(1-P^{2}\right)^{1 / 2}\left(1-K^{2}\right)^{-1 / 2}(1-K) J_{1}(1-K)^{-1}\left(1-K^{2}\right)^{1 / 2}\left(1-P^{2}\right)^{-1 / 2}(1-P)$.

Предложение доказано.

ЗАмЕЧАНИЕ. Из предложения следует результат, сформулированньй в теореме. Это позволяет дать положительный ответ на вопрос о существовании проекции $\mathscr{A}^{+}$на $\mathscr{A} \mathscr{O}_{g_{0}}^{+}$. Ответ на вопрос о сушествовании расслоения над $\mathscr{A}_{\omega}^{+}$, поставленньй выше, будет отрицательным. Это можно объяснить тем, что множество метрик, инвариантных относительно одной почти комплексной структуры, пересекается с множеством ассоциированных метрик не в одной точке. То есть одна и та же ПКС может быть ортогональной относительно нескольких ассоциированных метрик. Это не позволяет построить проекцию, указанную вьше.

\section{СПИСОК ЦИТИРОВАННОЙ ЛИТЕРАТУРЫ}

[1] Смоленщев Н.К. О пространстве римановых метрик на симплектическом и контактном многообразии // Сиб. матем. ж. 2001. Т. 42. №6. С. 1402-1407.

[2] McDuff D. Introduction to symplectic topology // IAS/Park City Math. Ser. 1999. V. 7. P. 8-33.

[3] Smolentsev N.K. The space of associated metrics on a symplectic manifold // E-print math.DG/0108110, 2001.

[4] Смоленщев Н. К. О кривизне пространства ассоциированных метрик на симплектическом многообразии // Сиб. матем. ж. 1992. Т. 33. №1. С. 132-139.

[5] Кобаяси Ш., Номидзу К. Основы дифференциальной геометрии. Ч. 1, 2. М.: Наука, 1981.

[6] Гантмахер Ф.Р. Теория матриц. М.: Наука, 1967. 